\title{
La evaluación estandarizada y el objetivo privatizador de gran escala: la polémica sobre el SIMCE y una mirada desde la visión de la economía
}

\author{
Osvaldo Jara Gómez*
}

\section{RESUMEN}

La Ley del Sistema de Aseguramiento de la Calidad (SAC) ha instalado con carácter legal y obligatorio la toma censal de pruebas estandarizadas a todos los establecimientos del sistema escolar chileno. El presente ensayo discute la forma en que los resultados de las pruebas estandarizadas, además, de informar a las familias sobre calidad de la educación de los colegios, podrían servir para informar sobre los flujos de caja de las instituciones a fin de permitir la evaluación de proyectos de inversión en gran escala en la industria educativa chilena.

Palabras clave: privatización de la educación, pruebas estandarizadas, industria educativa, aseguramiento de la calidad.

Standardized evaluation and the large-scale privatization purpose: The controversy about the SIMCE test and a perspective from the Economy \begin{abstract}
Quality Assurance System Law (SAC) has installed as legal and mandatory the census-like standardized tests to all centers of the Chilean school system. This paper discusses how the results of standardized tests also inform families about educational quality of schools and could be used to report cash flows of the institutions so as to allow the assessment of large scale investment projects in Chile's education industry.
\end{abstract}

Key words: privatization of education, standardized testing, education industry, quality assurance.

* Osvaldo Jara Gómez es M.A. Economía por la Georgetown University y estudiante del Doctorado en Educación de la Universidad de Playa Ancha (UPLA).E-mail: osvaldo.jara@gmail.com. El autor agradece muy especialmente al profesor Eduardo Cavieres por su rigurosidad, estímulo, compromiso y apoyo para la redacción final de este trabajo, además, de los comentarios a versiones iniciales de este artículo de las profesoras del Programa de Doctorado en la UPLA, María Angélica Oliva y Beatrice Ávalos. 


\section{Introducción}

Durante el año 2013, y por primera vez desde su creación en la década del '80, en Chile se expresó públicamente un rechazo hacia la aplicación de las pruebas estandarizadas del Sistema de Medición de la Calidad de la Educación (SIMCE). Así, un grupo de académicos (Alto al SIMCE, 2013) han puesto una alerta respecto de los dispositivos de evaluación del Sistema de Aseguramiento de la Calidad. Los académicos denunciaron las insuficiencias y deficiencias de usar el SIMCE como principal mecanismo de evaluación de la calidad en el sistema educativo.

En este contexto, el objetivo del presente ensayo es plantear una interpretación económica, que muestra la forma en que reducir el concepto de calidad a los resultados de las pruebas estandarizadas favorece la privatización del sistema escolar y crea otros escenarios de mayor profundización de la educación como un bien de mercado.

El texto está organizado en tres partes: la primera es una revisión de los argumentos presentados por el grupo de académicos respecto de las evaluaciones estandarizadas. La segunda parte discute algunas experiencias de EE.UU. y los efectos más recurrentes que se evidencian sobre el currículo y los roles de los profesores y los gestores de las escuelas. Esta evidencia complementa lo planteado por los académicos al indicar las consecuencias para Chile. En la tercera parte, se presenta una interpretación económica de cómo los efectos de la estandarización y la evaluación SIMCE son fundamentales para promover la privatización del sistema escolar, en tanto permiten construir indicadores homogéneos para estimar el valor económico de los establecimientos escolares en Chile. Ello permite sugerir algunas implicancias que esta privatización a partir de la estandarización tendrá en el futuro en el sistema educativo chileno, dada su tendencia a integrar modelos que provienen de países más desarrollados económicamente y en donde proveedores a gran escala de la industria educativa han tenido un sostenido crecimiento. 


\section{La discusión en Chile}

\subsection{Polémica sobre el SIMCE}

A propósito de las evaluaciones SIMCE, durante el año 2013 ocurrieron varios hechos destacables en Chile. El más relevante fue el que protagonizaron un grupo de reconocidos expertos sectoriales, incluyendo tres premios nacionales en educación, quienes cuestionaron técnicamente la Ley 20.529 aprobada el año 20I I, que creó el Sistema de Aseguramiento de la Calidad (SAC) (Ministerio de Educación, 20IIa), en específico, el rol del SIMCE como principal mecanismo para conocer la calidad de los establecimientos educacionales.

Los principales argumentos dados por estos expertos y publicados en la prensa a través de Alto al SIMCE (2013) son los siguientes:

i) Los resultados de las pruebas SIMCE reducen la complejidad de lo que significa educar.

ii) El SIMCE ha arrastrado a las comunidades escolares a destinar gran parte de sus esfuerzos a responder satisfactoriamente esta prueba.

iii) El SIMCE conlleva un creciente estrés y malestar en el profesorado y el estudiantado, afectando las relaciones en las comunidades escolares que año a año deben lidiar con esta prueba.

iv) El SIMCE individualiza a las escuelas públicamente y es usado por los proveedores para hacer propaganda sobre la calidad.

En base a estos argumentos, los citados académicos se plantean en oposición a: la aplicación censal y anual de las pruebas; el uso de los resultados para individualizar públicamente a las escuelas; la vinculación de los datos con incentivos a los establecimientos o sus actores; la reducción del currículo que el SIMCE provoca y la identificación de los resultados con la medición de calidad de la educación (Alto al SIMCE, 2013).

\subsection{Una tardía discusión sobre la Ley del SAC}

Si bien el SIMCE existe desde el año I988 (Bravo, 20II), y siempre ha tenido detractores y defensores, ¿por qué ahora esta discusión es tan relevante? A partir de la Ley del Sistema de Aseguramiento de la 
Calidad (SAC) se otorga una importancia inédita a la prueba SIMCE en el sistema escolar. Para ilustrar este problema en su amplia dimensión, se debe considerar que el resultado del SIMCE de este año determina en un $67 \%$ la clasificación oficial de la calidad de la educación de un colegio, tal como lo establece el Título II, Párrafo 3, Artículo I8 de la Ley. Cualesquiera otros elementos que determinen la calidad, tales como los aprendizajes en otras asignaturas o disciplinas, el nivel de inclusión de la escuela, la importancia de la escuela en el barrio donde se ubica, el compromiso del cuerpo docente y directivo con los estudiantes con mayores dificultades de aprendizajes; en definitiva, todo lo que no sea medido con el SIMCE, a lo sumo pondera un 33\%. Con los resultados del SIMCE, la Agencia de Calidad elabora un ordenamiento de establecimientos (un ranking). Según esto, se espera que las familias premien a los colegios de buena calidad y emigren desde los colegios de mala calidad.

La importancia de este tema salta a la discusión recién dos años después de haberse aprobado la Ley del SAC. El ordenamiento de establecimientos surge de una indicación del Ejecutivo en 20I I y que fue aprobada por la Comisión de Educación de la Cámara de Diputados, luego, por la Comisión Mixta y las dos salas con amplias mayorías (Congreso Nacional de Chile, 20I I). Por cierto llama la atención que durante la discusión en el Congreso no haya tenido suficiente notoriedad.

\section{2. ¿Qué podemos aprender de la experiencia comparada?}

Diversas investigaciones se han estado desarrollando en el mundo sobre las evaluaciones estandarizadas, especialmente en los países de la Organización para la Cooperación y el Desarrollo Económico (OECD) que realiza la prueba PISA (Angulo, 2009). Específicamente, este fenómeno en EE.UU. contiene una gran similitud con el caso chileno, dado que ambos países no solo aplican PISA, sino que cada año realizan evaluaciones estandarizadas e informan públicamente sus resultados. Además, las pruebas estandarizadas evalúan, principalmente, los subsectores de Lenguaje y Matemática y los resultados de estas generan consecuencias sobre la gestión de los establecimientos escolares. 


\subsection{El caso de Estados Unidos}

Los siguientes son efectos de gran relevancia observados con la Reforma en EE.UU., denominada "No Child Left Bebind" (NCLB), del año 2002 (United States Goverment, 2002).

a) Enseñar para las pruebas

Un primer efecto es que el currículum se intensifica, aumentándose las horas en las áreas medidas por la prueba y reduciéndose las horas de las áreas no medidas (Ravitch, 2009). Este proceso ha sido profusamente estudiado en Estados Unidos, llegándose a describir los incentivos perversos que la Ley NCLB produce a través del slogan "Teaching for the Test". Diane Ravitch, ex asesora del Gobierno Federal de EE.UU., ha aportado evidencias respecto de los problemas que esta Ley ha provocado en el sistema educacional (Ravitch, 2009), lo que ha representado un cuestionamiento general de los principales componente de la legislación (Lemann, 2008).

b) Incentivos pecuniarios que promueven conductas reñidas con la ética educativa y el fraude al sistema

Tal como lo ha señalado Stephenson (2006) respecto a EE.UU., introducir incentivos para mejorar los puntajes de los alumnos en pruebas estandarizadas lleva a que las instituciones educativas tiendan a adoptar conductas no éticas, incluso, fuera del marco legal. Por ejemplo, se ha reportado de escuelas que tratan de ocultar a los estudiantes rezagados para que no sean sometidos a las pruebas, desprestigiándolas, y a sus autoridades frente a la comunidad. El establecimiento de importantes premios pecuniarios a docentes, directivos y sostenedores, hace que los dispositivos de control deban especializarse en la fiscalización de la evasión o de la elusión de todos aquellos elementos que tienden a bajar los resultados y a falsear aquellos que tienden a subirlos (dentro o fuera de la ley).

c) Anulación de conductas colaborativas por la introducción de incentivos en forma individual a profesores $y$ directivos

A partir de los trabajos de Ravitch para EE.UU. (2009), se asocia los resultados de las pruebas estandarizadas de los alumnos a la 
calidad de los profesores de modo individual. Según la autora uno de los principales problemas es que se están eliminando los incentivos colectivos y colaborativos con los que tradicionalmente han funcionado las instituciones escolares.

\subsection{El caso de Chile}

En el caso de Chile, no existen investigaciones suficientes que permitan demostrar la ocurrencia de los fenómenos citados para la realidad norteamericana. No obstante, la propia Ley del SAC promueve que las instituciones dediquen gran parte de sus esfuerzos a los contenidos que el SIMCE evaluará. Por ejemplo, en II Medio, las materias vistas en Matemática y Lenguaje (las únicas que controla el SIMCE en este nivel) equivalen a dos tercios de toda la evaluación de la calidad que recibirá un Liceo en Enseñanza Media, lo que está explícito en la Ley 20.529, Título II, Párrafo 3, Artículo I8 (Ministerio de Educación, 20I Ia). De ello se derivan algunas consecuencias:

\section{a) Enseñar para el SIMCE}

El enseñar para la prueba SIMCE no solo es un fenómeno que ocurre en las instituciones por motivaciones consistentes con los incentivos de los resultados del SIMCE, sino que además es promovida como política por el propio Ministerio de Educación. Por ejemplo, el Plan de Apoyo Compartido del MINEDUC (201I), conocido como PAC, es precisamente un Programa cuyo objetivo principal es focalizar los esfuerzos pedagógicos en las materias que el SIMCE evalúa (cuadro I). Desde el punto de vista de la reducción curricular, el reciente estudio de Manzi et al. (2014) de la Universidad Católica, muestra que el $97 \%$ de los directivos de las escuelas hace ensayos de medición previos. También, establece que el 80,3\% de los directores adecúa las evaluaciones de sus establecimientos para que se asimilen al SIMCE y que el 78,4\% hace clases de reforzamiento para preparar el SIMCE. 
CUADRO I:

PLAN APOYO COMPARTIDO-RESUMEN DEL PLAN

Diseño y desarrollo de las herramientas de apoyo centralizado.

Los recursos pedagógicos para apoyar el fortalecimiento de competencias en la escuela son desarrollados por el Nivel Central del Ministerio de Educación, estos son principalmente:

- Planificación anual, periódica y plan de clases diario de NTI- $4^{\circ}$ Básico.

- Cuaderno de trabajo para el estudiante.

- Actividades niveladas para atender diversos ritmos de aprendizaje en el aula.

- Evaluación diagnóstica, de período y de final de año.

- Pauta de observación de clases diaria y periódica.

- Guías de reflexión técnica pedagógica, para que el equipo de liderazgo educativo pueda desarrollar reuniones con el equipo docente.

Fuente: www.mineduc.cl

b) Incentivos pecuniarios que promueven conductas reñidas con la ética educativa o fraudulenta

En tiempos recientes, se han publicado algunas denuncias de padres y apoderados, reclamando porque a sus pupilos no se les ha permitido dar la prueba SIMCE, debido a que el colegio o los profesores estiman que el promedio del SIMCE bajará si estos alumnos rinden el examen. La siguiente es una situación concreta de discriminación de una alumna de $\mathrm{I} 0$ años, aparecido en un periódico de circulación nacional en Chile precisamente en el día de la prueba SIMCE:

La empeñosa Kim P.V., de I0 años, le puso ene pino a las materias por estudiar, se quemó las pestañas varias semanas para quedar tiqui-taca y dar una excelente prueba Simce en su escuela, la Eleuterio Ramírez, del cerro Rocuant, en Valpo. Pero, según denuncia su familia, un profe 'fondeó a la peque y un compañerito pa' no perjudicar el desempeño del colegio. El miércoles pasado llegó a la escuela, se formó y antes de ingresar a su aula, se le acercó un docente y le dijo -según denuncia su familia- que ella y otro alumno estarían en otra sala para rendir la prueba. La niña na' de lesa cachó altiro el engaño y preguntó por qué la trasladaban, a lo que le respondieron que no se preocupara, que estaría en un lugar VIP.

Cerca de dos horas estuvo en la habitación con su compañero y vigilados por un profe, el cual no los dejó salir ni al pipiroom. 
Cerca del mediodía la liberaron, pero ya sus compañeros habían rendido la tan esperada Simce. Así que aguantando las lágrimas se fue a su casa en el sector Sor Teresa, en Cuesta Colorada (Henríquez, 2012, s.n.).

Unido a lo anterior, se encuentra el fenómeno de la selección. Aunque está legalmente prohibida la selección en los colegios subvencionados hasta $6^{\circ}$ Básico, se sabe que ciertos colegios particulares filtran a la entrada para evitar bajar sus puntajes SIMCE. Este aspecto es ilustrado por la declaración de un Colegio respecto a la adopción reciente de una nueva política de admisión:

Nos hemos reunido, como equipo de gestión, para analizar los requisitos que pediremos en el próximo proceso de postulación. Esto, en función de las directrices definidas por las nuevas autoridades en Educación. (...) Tenemos que bajar de la web la ficha de postulación para actualizarla, agregaron.

De esa manera, las autoridades del Colegio Gabriela Mistral (de Quinta Normal) informaron que ya están trabajando en modificar los requisitos de postulación a ese establecimiento particular subvencionado, en cuya ficha -hasta ahora-se pedían antecedentes médicos del alumno, conocer si él o su familia tenían ascendencia indígena o ficha de protección social.

El plantel es uno de más de una docena de colegios que hace dos meses pedían insólitas exigencias y documentos en su proceso de admisión, lo que está al margen de la ley, tal como lo reveló un reportaje de "La Segunda" publicado en marzo (De Améstica, 20I4: s.n.).

\section{c) Movimiento social contra la prueba SIMCE}

Hacia fines del 2013, también, se observó que un grupo de estudiantes de varios liceos municipales instalaron un movimiento de desobediencia civil, llamando a no rendir la prueba SIMCE. El resultado fue que en nueve liceos no se rindió la prueba o tuvo muy baja participación, según informó la Agencia de la Calidad (Estudiantes Instituto Superior de Comercio No 2, 2013). 


\subsection{EE.UU. y Chile en la ruta de la privatización}

La Ley NCLB (2003) establece que luego de tres evaluaciones deficientes el Gobierno de EE.UU. puede cerrar esas escuelas y traspasar los recursos hacia algún tipo de escuela subvencionada, charter scbool. El cierre de la escuela pública y la consecuencia posterior es un acto de política pública que reorganiza el sistema educativo. En el caso de Chile, la privatización ha ido ocurriendo lentamente a través de las decisiones individuales que las familias van adoptando año a año. La profundidad de la privatización de la educación en Chile tiene que ver con que es un proceso que ocurre en total armonía con las decisiones de cada una de las familias que van dejando atrás a la escuela municipal para tomar la opción del colegio particular subvencionado.

\section{Transformación del sistema educativo en un gran mercado Chile: Interpretación económica}

Los resultados de las pruebas SIMCE generan indicadores que pueden ser ocupados para hacer comparaciones entre colegios, para evaluar su cuerpo docente y sus alumnos y las familias de estos. Desde el punto de vista puramente económico, para algunas familias (consumidores de educación) estaría ocurriendo el siguiente fenómeno: i) el sistema les ofrece una serie de servicios entre los cuales están los resultados de aprendizajes (evidencia de esos resultados son los puntajes de las pruebas estandarizadas en las materias consultadas en el sistema SIMCE); ii) esta información es sintetizada en el ranking u ordenamiento del colegio; iii) la familia puede hacer un juicio de calidad fundado en indicadores oficiales; y iv) finalmente, según esto la familia puede asignar su voucher o subvención escolar al proveedor.

\subsection{La información que entrega el SIMCE y el ordenamiento}

La propuesta de la Ley del SAC es declarar que la calidad educativa de un colegio puede ser reflejada perfectamente por el ranking calculado en base a los resultados SIMCE, entonces, la familia estaría tomando 
una decisión con plena información. El ranking, por lo tanto, permite que las personas logren entender mejor esta información, pudiendo así ejercer su función de consumidores y destinar los recursos de la subvención al proveedor que mejor se ajuste a sus opciones.

En la práctica, esta estrategia ha funcionado de acuerdo a lo previsto y, apoyado por el SIMCE y sus resultados, el sistema se ha ido privatizando a lo largo de las últimas tres décadas. En efecto, los establecimientos municipales a nivel de todo el país han ido perdiendo matrícula, llegando a tener un total de $39 \%$ en 201 I; mientras que los establecimientos particular subvencionados la han ido ganando al ritmo inverso de los municipales, escalando a un total del 52\% en el mismo año (Ministerio de Educación, 20IIb).

Tradicionalmente, la educación ha sido entendida como un servicio que se produce con una gran diversidad de estilos, procesos y formas, tanto por el lado de los elementos que se utilizan en su producción (distintos tipos de insumos, distintos tipos de docentes, distintos modelos de gestión pedagógica), así como también genera distintos tipos de output, esto es, conocimientos y habilidades que son desarrolladas en las instituciones escolares. La estandarización rompe con esta dinámica diversa e induce la adopción de proyectos educativos estandarizados. Dicha lógica, junto con la estandarización de la evaluación y el ranking como único indicador de calidad, permite entregar más competitividad al mercado, por la vía de posibilitar una supuesta comparación entre establecimientos escolares. En la medida que las pruebas estandarizadas regulen y nivelen la actividad curricular según los estándares que las pruebas exigen, la población podría identificar muy claramente los niveles de calidad que alcanza su proveedor elegido.

\subsection{Implicancias de la estandarización desde la perspectiva económica}

La estandarización de la educación no tanto solo permite clasificar los establecimientos, posibilitando modos distintos de administración (privado o público), sino que también tiene múltiples implicancias en términos de la conformación de un mercado educativo. Los 
siguientes puntos exploran algunas de estas implicancias que, aunque no han sido abordadas aún en el debate sobre la privatización en Chile, son posibilidades latentes, considerando la tendencia del sistema educativo a importar modelos de países con mayor desarrollo económico.

a) La estandarización facilita las transacciones en el mercado, permitiendo el desarrollo de grandes empresas en un sistema predominantemente de pequeñas y medianas empresas

Si se asume el punto de vista del desarrollo de la educación como industria, se puede afirmar que la evaluación estandarizada y el ranking son las señales que permiten que los eventuales inversionistas evalúen el desarrollo de proyectos educacionales, ya sea de nuevos proyectos o para adquirir proyectos ya existentes, en escalas pequeñas o en gran escala. Para un inversionista, los resultados de las pruebas estandarizadas permiten tener un muy buen proxi de la estabilidad de los ingresos por matrículas.

\section{b) La estandarización facilita nuevas modalidades de escolarización}

Desde una perspectiva similar a la anterior, la reducción del currículo a los contenidos de las pruebas SIMCE hace posible la instalación de la industria educativa en modalidad escolaridad en casa o como se le denomina en inglés, Home Schooling. Esta forma educativa, que está exhibiendo un fuerte desarrollo en EE.UU. (Apple, 200I), opera bajo la misma figura de los charter school, pues se sustenta en emprendimientos de grandes empresas, que como lo plantea Ravitch (2013) se encuentran asociados a la industria editorial de materiales educativos y del software para la educación a distancia. De hecho, Ravitch (2013) identifica esto, mostrando que uno de los principales interesados en la estandarización es la Fundación de Bill y Melinda Gates.

a) La estandarización facilita transformar en bienes de consumo el sistema educativo a nivel global para capturar una importante porción del PIB mundial

Otra implicancia viene del ámbito de los negocios. Por ejemplo, en los países de la OECD en el año 201 I la participación del sector Educación como porcentaje del PIB fue de 6,3\%, lo que define un 
gran volumen de recursos privatizables a la hora de aplicar políticas de tinte neoliberal. En las últimas décadas, varios países con tradicionales sistemas estatales de educación (como EE.UU) han comenzado procesos de privatización de los servicios escolares. Por su parte, Chile fue un adelantado y ya en la década del '80 inició un proceso de integración de actores privados, con fines de lucro, a la provisión de educación financiada con fondos públicos.

\section{Comentario final}

De acuerdo a lo planteado en este ensayo, la estandarización puede ser entendida como la simplificación necesaria para interesar e introducir la provisión de servicios educacionales a escala por parte de corporaciones privadas con fines de lucro. La instalación de los dispositivos de estandarización facilitan que el establecimiento de las condiciones para que grandes empresas educacionales entren al mercado a hacerse cargo de la correspondiente administración de la participación en el PIB del sistema escolar a nivel nacional y, también, a nivel mundial. De esta manera, si bien es cierto que temas tales como el término del co-pago o la gratuidad de la educación escolar son fundamentales para generar igualdad, no es menos cierto que sin una discusión en profundidad sobre los efectos de la estandarización no se podrá revertir en el sistema la tendencia hacia su privatización y, consecuentemente, hacia su segregación económica y social.

\section{Referencias bibliográficas}

Alto al SIMCE (2013). Carta abierta por un nuevo sistema de Evaluación Educacional. Recuperado de http://www.lanacion.cl/premiosnacionales-y-academicos-firman-carta-abierta-en-rechazo-al-simce/ noticias/2013-09-27/120718.html

Angulo, F. (2009). Evaluaciones estandarizadas una vuelta al pasado. Revista de Docencia, 38, 19-25.

Apple, M. (200I). ¿Podemos luchar contra el neoliberalismo y neoconservadurismo en educación? Revista de Docencia, I3, 4-I0.

Bravo, J. (20II). SIMCE: pasado, presente y futuro. Estudios Públicos, I23 (invierno), I89-2II. 
Congreso Nacional de Chile (201 I). Historia de la Ley $N^{\circ}$ 20.529. Recuperado de www.leychile.cl/Navegar/scripts/obtienearchivo?id...3/.../I/ HL20529...

De Améstica, C. (9 de mayo de 2014). Colegios subvencionados cambian sus procesos de admisión. Recuperado de http://www.lasegunda.com/ Noticias/Nacional/2014/05/933767/seleccion-escolar-colegiossubvencionados-cambian-sus-procesos-de-admision

Departamento de Educación EE.UU. (2003). Que ningún niño se quede atrás, guía para padres. Washington D.C.: U.S.Education Departament.

Estudiantes Instituto Superior de Comercio No 2. (20 de noviembre de 2013). Declaración. Recuperado de www.altoalsimce.org: https://www.facebook. com/altoalsimce?fref=ts

Henríquez, D. (I9 de octubre de 2012). Kim dice que la fondearon pa' no bajar en la SIMCE. Recuperado de http://www.lacuarta.com/noticias/ cronica/20II/I0/63-I I633I-9-kim-dice-que-la-fondearon-pa-nobajar-en-la-simce.shtml

Lemann, N. (2008). What no child left bebind left bebind. Recuperado de http:/ / www. washingtonmonthly.com/features/2008/0808.lemann.html

Manzi, J., Bogolasky, F., Gabriel, G., Grau, V. \& Volante, P. (20I4). Análisis sobre valoraciones, comprensión y uso del SIMCE por parte de directores escolares de establecimientos subvencionados. Estudios de Política Educativa, 2, II4-I44.

Ministerio de Educación. (20IIa). Ley 20.529. Recuperado de http://www. leychile.cl/Navegar?idNorma $=1028635 \& \mathrm{r}=\mathrm{I}$

(20IIb). Anuario. Recuperado de http://centroestudios. mineduc.cl $/$ index.php?t $=968 \mathrm{i}=28 \mathrm{cc}=20368 \mathrm{tm}=2$

Ravitch, D. (2009). Time to kill 'no child left behind'. Recuperado de http:/ / www. edweek.org/ew/articles/2009/06/04/33ravitch_ep.h28.html (2I03). Reign of error, the boax of the privatization movement and the danger to America's public schools. New York: Ramdom House.

Stephenson, E. (2006). Evading no child left behind act: State strategies and Federal Complicity. B.Y.U. Education and Law Journal, I57-I88.

United States Goverment (2002). No child left bebind act. Recuperado de bttp:// www2.ed.gov/nclb/landing.jbtml 